\title{
To determine the eficacy of DIPSI as a method to screen GDM
}

\author{
Rashmi K., Anusha G. K.* \\ Department of Obstetrics and Gynecology, Bangalore Medical College and Research Institute, Bangalore, Karnataka, \\ India
}

Received: 26 October 2016

Revised: 01 November 2016

Accepted: 03 November 2016

*Correspondence:

Dr. Anusha G. K.,

E-mail: dr.anushagk@gmail.com

Copyright: () the author(s), publisher and licensee Medip Academy. This is an open-access article distributed under the terms of the Creative Commons Attribution Non-Commercial License, which permits unrestricted non-commercial use, distribution, and reproduction in any medium, provided the original work is properly cited.

\begin{abstract}
Background: Gestational Diabetes Mellitus (GDM) is considered as a transient abnormality of glucose intolerance during pregnancy. Women with GDM and their children are at increased risk of future diabetes. The diagnosis of GDM gives us a unique opportunity in identifying individuals who will be benefited by early therapeutic intervention with diet, exercise and normalizing the weight to delay or possibly even prevent the onset of the disease. The objective of the study was to detect efficacy of Diabetes In Pregnancy Study Group of India (DIPSI) recommended $75 \mathrm{~g}$ oral glucose challenge test in screening and diagnosis of GDM.

Methods: 200 pregnant women attending antenatal clinic during 24-28 weeks of pregnancy were subjected to Diabetes In Pregnancy Study Group of India (DIPSI) recommended 75g oral glucose challenge test (OGCT) as a method to screen and diagnose GDM. All patients were given 75 grams of glucose irrespective of the meal and 2 hour venous blood sample was collected. Blood glucose was tested by GOD-POD (glucose oxidase peroxidise) method. Diagnosis of impaired glucose tolerance was made when plasma glucose of $\geq 120-140 \mathrm{mg} / \mathrm{dl}$ and diagnosis of GDM was made when the plasma glucose of $>140 \mathrm{mg} / \mathrm{dl}$. All of them were followed up till delivery. Descriptive statistics was used to calculate the mean and standard deviation to draw the conclusion of this study.

Results: Out of the 200 women subjected to DIPSI recommended 75 grams of OGCT $38 \%$, 40\% and $22 \%$ had normal, impaired and abnormal OGTT results, respectively. Age $\geq 25 \mathrm{yrs}$, family history of diabetes and BMI $\geq 25 \mathrm{~kg} / \mathrm{m} 2 \mathrm{are}$ the commonest risk factors for GDM in the study group.

Conclusions: Our study supports the concept of universal screening and the use of DIPSI recommended 75g OGCT for screening and diagnosis of GDM.
\end{abstract}

Keywords: DIPSI, GDM, OGCT

\section{INTRODUCTION}

Gestational Diabetes Mellitus (GDM) is defined as "carbohydrate intolerance of variable severity that is first diagnosed during present pregnancy. It does not exclude the possibility that glucose intolerance may have antedated the pregnancy" ${ }^{1,2}$ Pregnancy is associated with increasing insulin resistance that begins in mid-pregnancy and progresses through the third trimester. Insulin resistance is due to the insulin - desensitising effects of placental hormones. ${ }^{3}$ GDM is associated with adverse maternal and neonatal complications such as the peripartum risks of macrosomia, hyperbilirubinemia, operative delivery, shoulder dystocia and birth trauma and the higher incidence of childhood obesity and long term risk of type 2 diabetes in mother and offspring. ${ }^{4}$

The most common risk factors of GDM are obesity, older maternal age, past history of GDM, strong family history of diabetes, member of an ethnic group with a high prevalence of T2DM, polycystic ovary syndrome. ${ }^{5}$ Diabetes Mellitus is the most common medical disorder of pregnancy, although the prevalence of GDM is usually reported as 2 to $5 \%$ of pregnant women, it can be as high as $14 \%$ in high risk groups. ${ }^{1,2}$ There is an increasing trend of Diabetes noted globally. India is considered as the 
world diabetes capital and Indians are the vulnerable sufferers of this disease. ${ }^{6}$ As such Universal screening during pregnancy have become important in India. For this we need a simple procedure which is economical and feasible.

Over decades many workshops and conferences have been held to device proper testing protocols to diagnose GDM. The controversy concerning optimal strategy still continues for the detection and diagnosis of GDM and this has lead to a wide variance in screening practices worldwide. Various screening guidelines have been introduced depending upon the suitability of the test to the population, characteristics, cost and the screening accuracy.

In the Indian context, Dr. Seshiah proposed that screening is essential in all pregnant women as the Indian women have 11 fold increased risk of developing glucose intolerance during pregnancy. The current guidelines by DIPSI 2014 recommended that testing by $75 \mathrm{~g} 2 \mathrm{hr}$ oral glucose challenge test need not be done in fasting state and is done irrespective of the last meal. ${ }^{7}$ This prospective study was undertaken to ascertain the validity of the DIPSI criterion in diagnosing GDM.

\section{METHODS}

This was a prospective observational study conducted on a total number of 200 pregnant women in the department of Obstetrics and Gynaecology for a period of 2 years. All pregnant women attending antenatal clinic during 24 28 weeks of pregnancy were included in the study; excluding those who were pre-gestational diabetics. The procedure of the study was explained and required consent for the study was taken. A detailed history and examination was undertaken and all relevant data was obtained. FBS and $\mathrm{HbA} 1 \mathrm{C}$ blood tests were done at the initial antenatal visit to rule of women with pre-existing diabetes mellitus. All patients were given 75 grams of glucose irrespective of the meal and 2 hour venous blood sample was collected. Blood glucose was tested by GODPOD (glucose oxidase peroxidise) method. Diagnosis of impaired glucose tolerance was made when plasma glucose of $\geq 120-140 \mathrm{mg} / \mathrm{dL}$ and diagnosis of GDM was made when the plasma glucose of $>140 \mathrm{mg} / \mathrm{dL}$. Women diagnosed as GDM were managed appropriately. All of them were followed up until delivery. Descriptive statistics was used to calculate the mean and standard deviation to draw the conclusion of this study.

\section{RESULTS}

A total of 200 antenatal pregnant women aged between 19 to 35 years comprising of 120 multigravida and 80 primigravida were studied. These women were subjected to DIPSI recommended 75 grams of OGCT at 2428weeks of gestation and were followed up until delivery. The mean age of study population was $23.65 \pm 3.61$ years. Of the 200 women subjected to DIPSI recommended 75 grams of OGTT $38 \%, 40 \%$ and $22 \%$ had normal, impaired and abnormal OGCT results, respectively (Table 1$)$.

Table 1: Distribution of study sample based on the OGCT values $(n=200)$.

\begin{tabular}{|c|c|c|c|}
\hline \multirow{2}{*}{ Final diagnosis } & \multicolumn{2}{|c|}{ DIPSI method } & \multirow{2}{*}{ p value } \\
\hline & No & $\%$ & \\
\hline Normal & 76 & 38 & $<0.001 * *$ \\
\hline Impaired GT & 80 & 40 & $<0.001 * *$ \\
\hline Deranged/abnormal & 44 & 22 & 0.220 \\
\hline Total & 200 & 100 & - \\
\hline
\end{tabular}

$\mathrm{p}<0.001^{* *}$, significant, Fisher Exact test

Out of 200 patients in DIPSI study group 38\% had no GDM, whereas $22 \%$ had abnormal glucose tolerance and $40 \%$ had impaired glucose challenge test result. This test showed strongly significant $\mathrm{p}$ value of $<0.001$. This proves that DIPSI method detected more number of cases with GDM.

The commonest risk factors for GDM in the study group are age $\geq 25 \mathrm{yrs}(35 \%)$, family history of diabetes $(34 \%)$ and BMI $\geq 25 \mathrm{~kg} / \mathrm{m}^{2}$ (47\%). This showed that $58 \%$ had risk factors for GDM in the present pregnancy and $42 \%$ did not have any risk factors in the present pregnant. Among the $62 \%$ women with abnormal glucose challenge test, $38 \%$ were treated with diet and $24 \%$ required insulin. Most common mode of delivery observed in the study population was normal delivery and Caesarean delivery accounting to $47 \%$, with common indication being previous Caesarean delivery and foetal distress, $2 \%$ had instrumental delivery due to $2^{\text {nd }}$ stage complications and $2 \%$ had preterm deliveries due to PROM. Maximum weight of babies was between 2.5$3.5 \mathrm{~kg}$ group-70\%, $21 \%$ was observed in $1.5-2.4 \mathrm{~kg}$ group and $9 \%$ was observed in $>3.5 \mathrm{~kg}$ group.

\section{DISCUSSION}

In ancient times Diabetes in pregnancy was considered a complication of pregnancy, incompatible with life, and therefore, pregnancy was not allowed in women with diabetes. ${ }^{8}$ Clinical recognition of GDM is important because therapy, including diet, insulin when indicated and antepartum foetal surveillance can reduce the perinatal morbidity and mortality. With the advent of experimentation and groundwork in this field many developments have come up in managing Diabetes in pregnancy. Yet there is no universal agreement on the screening strategies and diagnostic criteria of GDM. ${ }^{9}$

The Diabetes in Pregnancy Study group India (DIPSI) has reported practice guidelines for GDM in the Indian environment. Due to high prevalence, screening is essential for all Indian pregnant women. DIPSI guideline is "a one step procedure with a single glycemic value", to diagnose GDM in the community. It is ideal for resourcechallenged settings like India. It is a single step method which is easier and convenient to patients as they do not 
need to fast. According to the DIPSI group the rationale of performing this type of OGTT is that after a meal, a normal glucose tolerant woman would be able to maintain euglycemia despite the glucose challenge due to a brisk and adequate insulin release. In the case of a pregnant woman who has impaired insulin secretion, she will not be able to maintain euglycemia after a meal and there will be a further increase in plasma glucose value after an OGTT. This cascading effect is advantageous and would not result in false positive diagnosis of GDM. ${ }^{10}$

In this study among the 200 healthy pregnant women followed up during pregnancy $38 \%, 40 \%$ and $22 \%$ had normal, impaired and abnormal OGCT results respectively with DIPSI recommended 75 grams of OGCT irrespective of the last meal taken. Our study result is comparable to V. Bajaji et al study. ${ }^{6}$ In Seshiah et al study it was observed that age $\geq 25 \mathrm{yrs}$, BMI $\geq 25 \mathrm{~kg} / \mathrm{m} 2$ and family history of diabetes were significantly associated with the prevalence of GDM. ${ }^{7}$ Our study can be compared with Seshiah et al for prevalence of risk factors which proves that majority of Asian women are at a high risk to develop GDM. The trend of decline in perinatal and neonatal outcome noted in the study may reflect improvement in general health awareness and better availability and utilization of health care facilities.

\section{CONCLUSION}

The prevalence of GDM is increasing from $2 \%$ in 1982 to $16.55 \%$ in 2001 with previously followed criteria for diagnosis of GDM, whereas using DIPSI 2010 for diagnosis of GDM, we were able to detect GDM among $57 \%$ of antenatal mother showing the efficacy of DIPSI on higher side. Amongst risk factors, family history of diabetes mellitus, age and BMI are the most important risk factors for increasing prevalence. ${ }^{10}$ Our study supports the concept of universal screening and the use of $75 \mathrm{~g}$ oral glucose challenge test with a cut-off value of $120-140 \mathrm{mg} / \mathrm{dl}$ for impaired result and $\geq 140 \mathrm{mg} / \mathrm{dl}$ for deranged or abnormal result for glucose intolerance and screening and diagnosis of GDM by the DIPSI method which is practical and cost effective approach in the diagnosis of GDM.

\section{ACKNOWLEDGEMENT}

We express our heartfelt thanks to the Dean and Director, all the staff and postgraduate colleagues of the Department of Obstetrics and Gynaecology and all the pregnant mothers who have supported us for the study.
Funding: No funding sources Conflict of interest: None declared

Ethical approval: The study was approved by the Institutional Ethics Committee

\section{REFERENCES}

1. ACOG Practice Bulletin, Clinical management guidelines for obstetrician-gynaecologists. Number 30, (replaces technical bulletin number 200, December 1994) Gestational diabetes. Obstet Gynecol, 2001;98(3):525-38.

2. Berger H. Screening for gestational diabetes mellitus. J Obstet Gynaecol Canada. 2002;24(11):894-912.

3. Udipi VB. Effectiveness of Diabetes in Pregnancy Study Group India (DIPSI) Diagnostic Criterion in Detecting Gestational. Indian Journal of Basic and Applied Medical Research. 2013;2(6):614-8.

4. Vambergue, Brody SC, Harris R, Lohr. Diabetes Mellitus-a Pilot Study in a Rural Population. Screening of gestational diabetes: a summary of the evidence for the U. S. Preventive Series Task Force. Obstet Gynecol 2003;101(2):380-92.

5. Global Guideline- Pregnancy and Diabetes, IDF Guidelines. 2009.

6. Balaji V, Balaji M, Anjalakshi C, Cynthia A, Arthi T, Seshiah V. Diagnosis of gestational diabetes mellitus in Asian-Indian women. Indian J Endocrinol Metab. 2011;15(3):187-90.

7. Seshiah V, Balaji V, Balaji MS, Paneerselvam A, Arthi T, Thamizharasi M, et al, Article J Assoc Physicians India. 2008;56:329-33. (Accessed online on 24/4/12

at www.ncbi.nlm.nih.gov/pubmed/18700640.

8. Hadden DR. A Historical Perspective on Gestational Diabetes. From the Fourth International WorkshopConference on Gestational Diabetes Mellitus. Diabetes Care 1998;21(2):B3-4.

9. Gupta S. Gestational Diabetes Mellitus (GDM): We Need to Revise the Standard Criteria for Diagnosis: Indian Experience. Australian Diabetes in Pregnancy Group (ADIP) meet: Sydney; 2004.

10. Seshaiah V. Fifth national conference of diabetes in pregnancy study group in India. J Assoc Physicians India 2010;58:329-30.

11. Purandare $\mathrm{CN}$. Universal screening for gestational diabetes mellitus (GDM): mandatory. J Obstet Gynecol India. 2012;62:141-3.

Cite this article as: Rashmi K, Anusha GK. To determine the eficacy of DIPSI as a method to screen GDM. Int J Reprod Contracept Obstet Gynecol 2016;5:4193-5. 